\title{
Modification of Nonlinear Optical Properties in CdSe/PBMA Film by High Intensity Light
}

\author{
V. Kovalevskij ${ }^{a, *}$ And V. Gulbinas ${ }^{a, b}$ \\ ${ }^{a}$ Institute of Physics, Savanoriu pr. 231, 02300 Vilnius, Lithuania \\ ${ }^{b}$ Vilnius University, Sauletekio al. 9, bldg 3. 2040 Vilnius, Lithuania
}

Excitation-relaxation dynamics in CdSe nanocrystals passivated with tri- $n$-octylphosphine oxide and embedded in the poly(butylmethacrylate) polymer was investigated before and after their irradiation by high intensity laser pulses. The irradiation causes a significant bleaching of the sample absorption as well as the shift of the lowest energy absorption band to the short wavelength side. The film irradiation reduces the nonlinear transient absorption bleaching on the long wavelength side of the low energy absorption band, however, it has only minor influence on the transient absorption relaxation.

PACS numbers: 78.67.-n

\section{Introduction}

Achievements in the refinements of the preparation of highly monodisperse colloidal nanocrystals ( $\mathrm{NCs}$ ) have actuated numerous spectroscopic studies in recent decades. They are basically motivated by the possibility of the future applications of NCs in optoelectronics and signal processing [1,2]. Because of the three-dimensional carrier confinement and a large surface-to-volume ratio, semiconductor nanocrystals exhibit a number of unusual physical properties which are not inherent in corresponding bulk semiconductors [1]. Possible applications can benefit not only from the size-controlled spectral tunability and the enhancement of the oscillator strength, but also from ultrafast relaxation dynamics, being largely controlled by the condition of the NC surface and environment [3-6].

*corresponding author; e-mail: vitalij.kovalevskij@ff.vu.lt 
The stability upon irradiation of NCs-based materials is one of the most important issues for their successful application. This is particular important for nonlinear optical devices where the active materials are exposed to the high intensity light.

In this paper we address the excitation relaxation dynamics in tri- $n$-octylphosphine oxide (TOPO) passivated CdSe NCs and embedded in poly(butylmethacrylate) (PBMA) polymer matrix, transparent in the visible region. We investigate how the transient absorption properties of the CdSe NCs doped polymer films alter after their irradiation by the high intensity laser light.

\section{Experimental}

CdSe nanocrystalline quantum dots were synthesized using the method established by Murray et al. [7]. After the synthesis of NCs, passivated with TOPO, the size distribution was further narrowed by size-selective precipitation using methanol and toluene.

To prepare the polymer films, NCs dispersed in toluene were mixed with slightly polar PBMA polymer, also dissolved in toluene. The mixture was dropped on the quartz substrate and left to dry for a few days. We report results for $50 \AA$ mean diameter NCs that have an absorption maximum at $590 \mathrm{~nm}$ in toluene. Absorption spectra were measured using a Beckman UV-5270 absorption spectrophotometer.

The picosecond transient absorption (TA) study was performed by using a homemade pump-probe spectrometer based on a Nd:glass laser delivering pulses of 3 ps duration at $3 \mathrm{~Hz}$ repetition rate. The second harmonic of the fundamental light at $527 \mathrm{~nm}$ generated in a KTP crystal was used for the sample excitation.

The femtosecond TA was investigated by the fs pump-probe spectroscopy. The sample was excited at $400 \mathrm{~nm}$ by frequency-doubled pulses from a regeneratively amplified mode-locked Ti-sapphire laser. The laser generated pulses of about 100 fs duration at $1 \mathrm{kHz}$ repetition rate. The transmission of the excited sample was probed over the 480-700 $\mathrm{nm}$ range with variably delayed pulses from an fs continuum, generated in a $3 \mathrm{~mm}$ thick sapphire plate.

\section{Results and discussions}

Irradiation of the CdSe/PBMA polymer film by high intensity picosecond pulses causes clearly visible bleaching of the film absorption. Figure 1 shows absorption spectra of the film before and after its exposure to two thousand of 3 ps duration pulses at $527 \mathrm{~nm}$ with energy density of $600 \mu \mathrm{J} / \mathrm{mm}^{2}$. The film absorption decreases in the entire visible spectral region and the lowest energy absorption band corresponding to the $1 S(\mathrm{e})-1 S_{3 / 2}(\mathrm{~h})$ transition shifts to the short energy side by about $12 \mathrm{~nm}$. Figure 2 represents the transient absorption spectra 

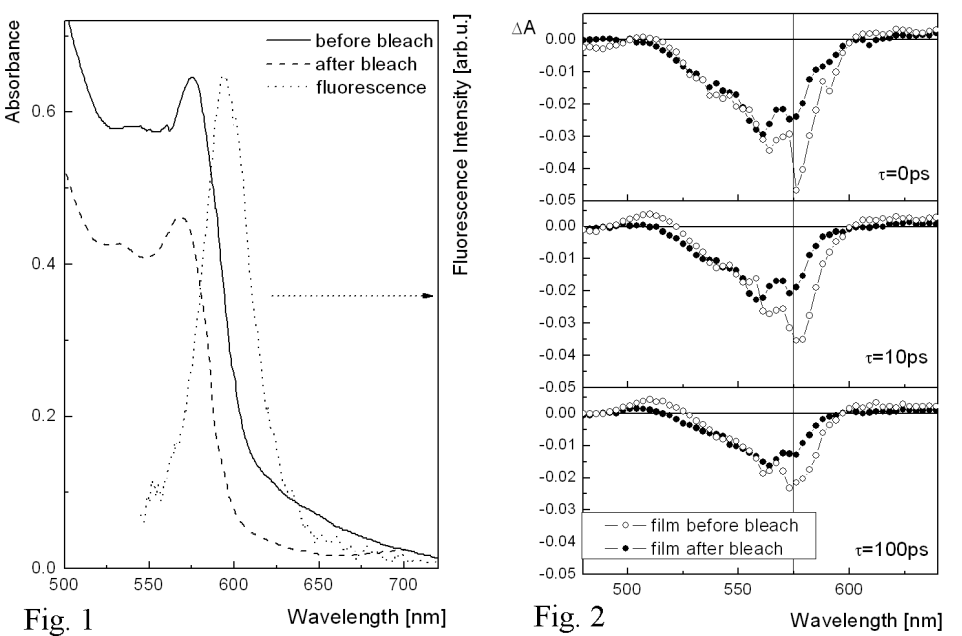

Fig. 1. Absorption (solid and dashed curves) and luminescence (dotted curve) spectra of TOPO-passivated $50 \AA$ mean diameter CdSe NCs embedded in the PBMA matrix. The luminescence intensity of this sample is given in arbitrary units.

Fig. 2. $\Delta A$ spectra of TOPO-passivated CdSe/PBMA NCs before (open circles) and after (solid circles) bleach recorded at various time delays after excitation at $400 \mathrm{~nm}$.

of fresh and exposed films after their excitation by femtosecond pulses at $400 \mathrm{~nm}$. Transient spectra show absorption bleaching of the low energy absorption band corresponding to the population of the lowest energy electron and hole states. The absorption bleaching decreases at longer times and a weak induced absorption appears at higher energies. The maximum of the bleaching band of the fresh sample corresponds to the maximum of the steady-state absorption. After sample irradiation, the transient differential absorption at the low energy absorption band slope decreases, and the maximum of the bleaching band shifts to the higher energy side. The induced absorption at longer delay times becomes also significantly weaker. However, the sample irradiation has no influence on the transient absorption at around $550 \mathrm{~nm}$, despite that the steady-state absorption decreases in this spectral region as well.

Figure 3 represents the intensity-dependent $\Delta A$ time evolution of the bleaching band measured at $575 \mathrm{~nm}$. Three processes could be distinguished during relaxation. The first process is characterized by a picosecond relaxation time of about 3-4 ps and is independent of the excitation intensity. According to Klimov et al. [5] it should be related to the surface induced quenching of electron-hole pairs. The intermediate relaxation is characterized by a 40 picosecond time constant. Although the corresponding relaxation component is almost negligible at very low excitation, it gains intensity by increasing excitation level at the expense of the slowest relaxation component, suggesting that the intermediate time relax- 

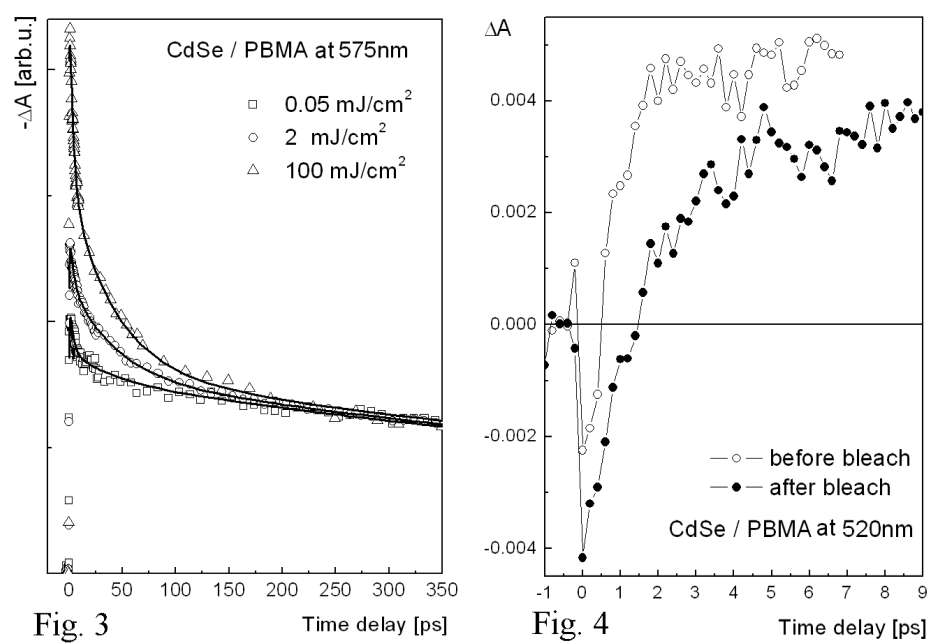

Fig. 3. Pump-dependent $\Delta A$ dynamics after excitation at $400 \mathrm{~nm}$ recorded at $575 \mathrm{~nm}$ for TOPO-passivated CdSe NCs embedded in PBMA polymers and normalized to the slowest process.

Fig. 4. Time dependence of the $\Delta A$ signal (symbols) recorded at $520 \mathrm{~nm}$ after excitation at $400 \mathrm{~nm}$ of TOPO-passivated CdSe/PBMA NCs recorded before and after bleach.

ation process is caused by the two or more electron-hole pairs created in the NC, which subsequently relax according to an Auger-type relaxation mechanism as proposed in Ref. [8]. The slowest relaxation component refers to the last electron-hole pair recombination taking place in the NC volume. Shapes of bleaching kinetics measured at $575 \mathrm{~nm}$ in fresh and exposed samples (not presented) are completely identical, only the amplitudes of all components are lower in accordance with the transient absorption spectra.

Transient absorption kinetics of fresh and exposed films near the isobestic point at $520 \mathrm{~nm}$ are slightly different (Fig. 4). During several ps, absorption bleaching at this wavelength is replaced by the induced absorption. After sample irradiation, the induced absorption becomes weaker, and it appears to be slightly slower.

All above presented experimental data enable us to conclude that the irradiation of the polymer film doped with CdSe NCs changes their linear absorption, as well as optical nonlinearities. Although the linear absorption is significantly bleached in the entire visible spectral region, the nonlinear optical properties of the film change less significantly. The sizeable reduction of the transient absorption is observed only on the long wavelength side of the lowest energy absorption band. The irradiation influence on the transient absorption kinetics is also observed only near the isobestic point where the transient absorption is very sensitive to the minor spectral changes. These observations suggest that the sample irradiation 
causes modification only of some particular fraction of CdSe NCs, which is responsible for a significant part of the light absorption, but its optical nonlinearities are weaker. Since the irradiation almost completely bleaches the film absorption at the long wavelength tail, it is evident that namely the largest NCs absorbing in this spectral region are dominantly modified. It is still difficult to tell if these NCs are completely destroyed by the high intensity light, or their size is reduced, so that their absorption shifts to the shorter wavelengths.

\section{Conclusions}

In conclusion, we have investigated modification by the high intensity laser pulses of the linear and differential transient absorption properties of CdSe NCs embedded in the PBMA polymer matrix. Although sample irradiation significantly changes the linear absorption, its influence on nonlinear optical properties is less dramatic. This suggests that light irradiation causes modification predominantly of the largest NCs absorbing on the long wavelength side of the absorption spectrum.

\section{Acknowledgments}

Authors are grateful to prof. G. Scholes for sample preparation and to colleagues from Vilnius University Laser Research Center for possibility to use femtosecond laser system.

\section{References}

[1] A.P. Alivisatos, J. Phys. Chem. 100, 13226 (1996).

[2] V. Klimov, P. Haring-Bolivar, H. Kurz, Phys. Rev. B 53, 1463 (1996).

[3] V. Kovalevskij, V. Gulbinas, A. Piskarskas, M.A. Hines, G.D. Scholes, Phys. Status Solidi B 241, 1986 (2004).

[4] C.A. Leatherdale, M.G. Bawendi, Phys. Rev. B 63, 165315 (2001).

[5] V.I. Klimov, D.W. McBranch, C.A. Leatherdale, M.G. Bawendi, Phys. Rev. B 60, 13740 (1999).

[6] N. Tessler, V. Medvedev, M. Kazes, S.-H. Kan, U. Banin, Science 295, 1506 (2002).

[7] C.B. Murray, D.J. Norris, M.G. Bawendi, J. Am. Chem. Soc. 115, 8706 (1993).

[8] V. Klimov, D.W. McBranch, Phys. Rev. B 55, 13173 (1997). 